Western University

Scholarship@Western

Brain and Mind Institute Researchers'

Publications

Brain and Mind Institute

$10-1-2015$

\title{
Maternal Postsecondary Education Associated With Improved Cerebellar Growth After Preterm Birth.
}

\author{
Mikaela L Stiver \\ Collaborative Neuroscience Program, University of Guelph, Guelph, Ontario, Canada \\ Daphne Kamino \\ Division of Neurology, Hospital for Sick Children, Toronto, Ontario, Canada \\ Ting Guo \\ Division of Neurology, Hospital for Sick Children, Toronto, Ontario, Canada \\ Angela Thompson \\ Division of Neurology, Hospital for Sick Children, Toronto, Ontario, Canada \\ Emma G Duerden \\ Division of Neurology, Hospital for Sick Children, Toronto, Ontario, Canada
}

Division of Neurology, Hospital for Sick Children, Toronto, Ontario, Canada Department of Psychology and

See next page for additional authors

Follow this and additional works at: https://ir.lib.uwo.ca/brainpub

Part of the Neurosciences Commons, and the Psychology Commons

\section{Citation of this paper:}

Stiver, Mikaela L; Kamino, Daphne; Guo, Ting; Thompson, Angela; Duerden, Emma G; Taylor, Margot J; and Tam, Emily W Y, "Maternal Postsecondary Education Associated With Improved Cerebellar Growth After Preterm Birth." (2015). Brain and Mind Institute Researchers' Publications. 171.

https://ir.lib.uwo.ca/brainpub/171 


\section{Authors}

Mikaela L Stiver, Daphne Kamino, Ting Guo, Angela Thompson, Emma G Duerden, Margot J Taylor, and Emily W Y Tam 


\title{
Maternal Postsecondary Education Associated With Improved Cerebellar Growth After Preterm Birth
}

\author{
Mikaela L. Stiver, BSc',2, Daphne Kamino, MSc', Ting Guo, PhD', \\ Angela Thompson, RN', Emma G. Duerden, PhD', Margot J. Taylor, PhD ${ }^{3,5}$, \\ and Emily W.Y. Tam, MDCM, MAS, FRCPC ${ }^{1,4}$
}

\begin{abstract}
The preterm cerebellum is vulnerable to impaired development impacting long-term outcome. Preterm newborns $(<32$ weeks $)$ underwent serial magnetic resonance imaging (MRI) scans. The association between parental education and cerebellar volume at each time point was assessed, adjusting for age at scan. In 26 infants, cerebellar volumes at term $(P=.00 \mathrm{I})$, but not birth $(P=.4)$, were associated with 2 -year volumes. For $\mathrm{I} \mathrm{cm}^{3}$ smaller cerebellar volume (4\% total volume) at term, the cerebellum was $3.18 \mathrm{~cm}^{3}$ smaller ( $3 \%$ total volume) by 2 years. Maternal postsecondary education was not associated with cerebellar volume at term $(P=.16)$. Maternal postsecondary education was a significant confounder in the relationship between term and 2 -year cerebellar volumes $(P=.016)$, with higher education associated with improved volumes by 2 years. Although preterm birth has been found to be associated with smaller cerebellar volumes at term, maternal postsecondary education is associated with improved growth detectable by 2 years.
\end{abstract}

\section{Keywords}

socioeconomic status, brain development, prematurity, cerebellar hypoplasia, magnetic resonance imaging (MRI)

Received October 17, 2014. Received revised January 13, 2015. Accepted for publication February 16, 2015.

Preterm birth is an important clinical issue, as it is linked to a range of prenatal and long-term difficulties, as well as distal factors including socioeconomic status. ${ }^{1}$ It is also frequent, with $11.7 \%$ of births being preterm in the United States in $2011{ }^{2}$ The high risk of neurological sequelae following preterm birth has stimulated interest in early brain development and growth, with a recent surge in interest in the cerebellum. The contributions of prenatal and distal factors on functional outcomes and brain development remain unclear. The cerebellum is particularly vulnerable to impaired development resulting from environmental factors after preterm birth as it undergoes exponential growth, both in volume and surface area, during the latter half of gestation. ${ }^{3-5}$ Cerebellar hypoplasia is associated with premature birth, especially in the presence of risk factors such as intraventricular hemorrhage $e^{6,7}$ and postnatal glucocorticoid exposure. ${ }^{8}$ The resultant reduction in cerebellar volume and compromised cerebellar development has been associated with cognitive and motor impairments. ${ }^{9}$

Many studies have documented correlations among aspects of socioeconomic status such as parental education, IQ, and scholastic achievement on neurodevelopmental outcome. ${ }^{10,11}$ Intellectual attainment has been found to be more strongly predicted by maternal education (cohorts of younger children) ${ }^{10}$ or equally by both parents' educations (cohorts of older children), ${ }^{12}$ depending on the age at which outcome was studied. In general, higher parental education and higher socioeconomic status are associated with stronger parenting skills and greater access to resources to aid in raising a child. ${ }^{13}$ Although previous research has shown correlations between both cerebellar volume $^{14,15}$ and socioeconomic status ${ }^{16-19}$ to neurodevelopmental outcome, the relationship between socioeconomic status and structural changes within the cerebellum has not been well studied. This investigation determined the association

\footnotetext{
' Division of Neurology, Hospital for Sick Children, Toronto, Ontario, Canada

${ }^{2}$ Department of Psychology and Collaborative Neuroscience Program, University of Guelph, Guelph, Ontario, Canada

${ }^{3}$ Department of Diagnostic Imaging, Hospital for Sick Children, Toronto, Ontario, Canada

${ }^{4}$ Department of Pediatrics, University of Toronto, Toronto, Ontario, Canada

${ }^{5}$ Department of Psychology, University of Toronto, Toronto, Ontario, Canada
}

\section{Corresponding Author:}

Emily W.Y. Tam, MDCM, MAS, FRCPC, Division of Neurology, Hospital for Sick Children, University of Toronto, 555 University Avenue, Toronto,

Ontario M5G IX8, Canada.

Email: emily.tam@utoronto.ca 


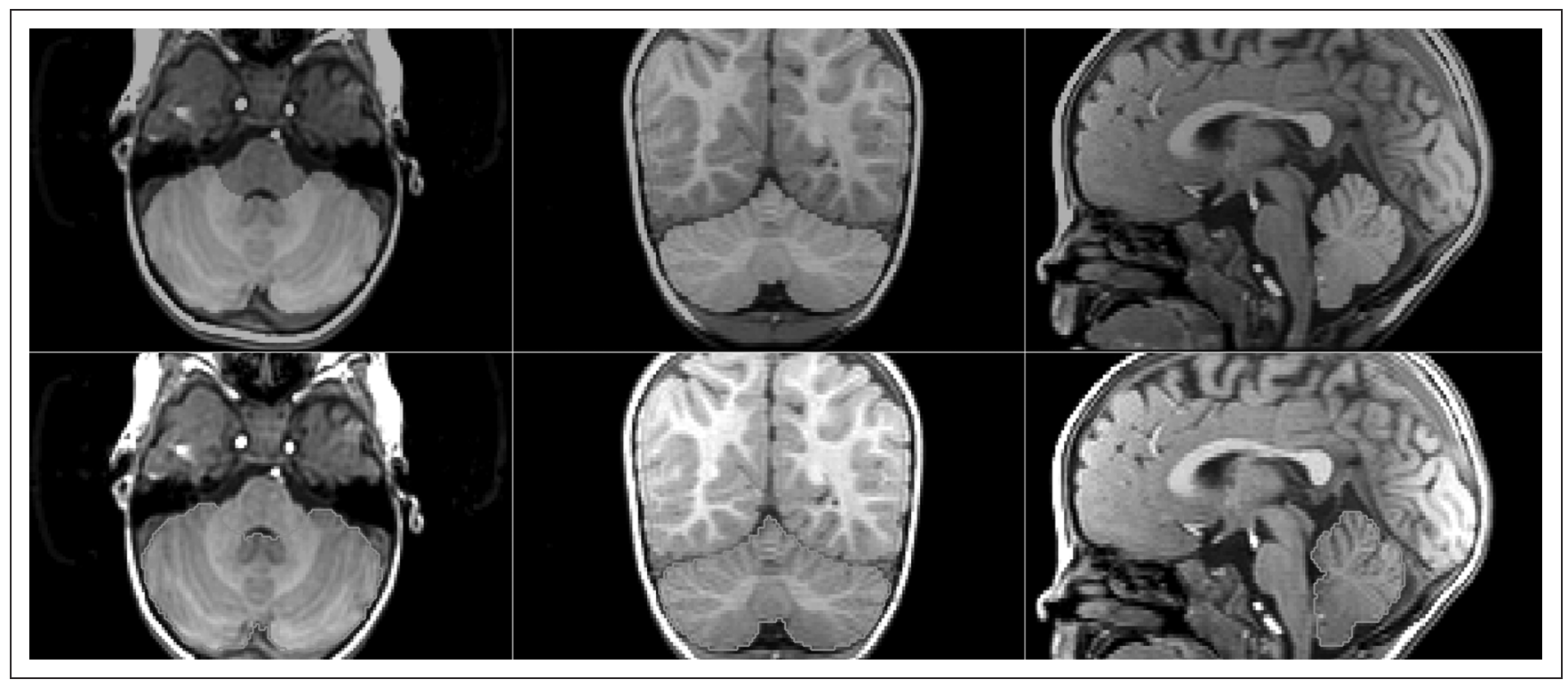

Figure I. Cerebellar volume at 2 years of age. Volumetric tracing of cerebellum superimposed on 3-dimensional TI-weighted magnetic resonance images (MRIs) obtained at I46 weeks + I day postmenstrual age. Images are shown in axial, coronal, and sagittal planes.

between changes in cerebellar volume (from early postnatal age to term-equivalent age, and then to 2 years) and socioeconomic status as represented by the level of parental education. Because correlations exist among outcomes, such as working memory, ${ }^{20}$ cognitive, ${ }^{21}$ and language-associated skills, ${ }^{22}$ to both physiological changes and socioeconomic status, we hypothesized that a positive correlation also exists between changes in cerebellar volume and parental education, as a measure of socioeconomic status.

\section{Methods}

\section{Study Subjects}

A cohort of very preterm infants born between January 2008 and August 2010 admitted to the NICU at the Hospital for Sick Children was recruited. Parents of very premature infants $(<32$ weeks gestational age) were approached in the first week of life for enrolment in a longitudinal MRI and neurodevelopmental outcome study. Exclusion criteria were (1) major congenital malformations, (2) chromosomal abnormalities, and (3) congenital infections. This study was approved by the Research Ethics Board at the Hospital for Sick Children, and parental consent was obtained for each infant. The infants included in the present study were neonates for whom a 2-year MRI scan and 2-year neurodevelopmental follow-up were completed. Developmental assessments were performed at 2 years corrected age using the Bayley Scales of Infant Development, 3rd Edition, scored in cognitive, language, and motor domains.

Clinical history was obtained from hospital medical records. Information was collected on the occurrence of clinical sepsis, and clinically presumed (maternal fever and tachycardia, with or without other direct evidence of fundal tenderness, purulence, or foul odor) and pathologically diagnosed chorioamnionitis (from placental pathology or bacterial culture). Postnatal exposure to glucocorticoids included hydrocortisone, dexamethasone, and cortisol. Data on maternal and paternal education were collected as indicators of socioeconomic status during the neurodevelopmental follow-up visits. Parents were asked to indicate their highest level of completed education: grade school, high school, postsecondary training, university, or postgraduate training. Body weight and head circumference of the children were also obtained at the time of the 2-year MRI scan.

\section{Neuroimaging Analysis}

MRI scans were performed with a 1.5-Tesla Signa Twin EXCITE3 scanner (General Electric Medical Systems, Milwaukee, WI; software rev.12M4) using an MRI-compatible incubator and small, specialized head coil (Advanced Imaging Research Incorporated, Cleveland, $\mathrm{OH}$ ) for the preterm and term-age scans to optimize subject comfort and monitoring, reduce movement, and improve signal-to-noise ratio. High-resolution axial 3-dimensional T1-weighted spoiled gradient recalled (SPGR) (repetition time, 23 milliseconds; echo time, 4 milliseconds; field of view, $128 \times 128 \mathrm{~mm}$; resolution, $1 \times 1 \times \mathrm{mm}$ ) and 2-dimensional T2-weighted fast recovery fast spin-echo (FRFSE) were run for each subject, as per previously published protocols. ${ }^{23}$ Subjects were scanned within 2 weeks of birth when clinically stable, at term-equivalent age, and at 2 years corrected age. The preterm and term infants were scanned in natural sleep, usually after feeding. The 2-year-olds were scanned in sleep as well, but this was facilitated in the majority with an oral sedative (chloral hydrate).

A neuroradiologist reviewed all MRI scans for severity of intraventricular hemorrhage, white matter injury, and cerebellar hemorrhage. Subjects were scored for the highest severity of injury detected on any of the serial scans.

The cerebellum was segmented on axial 3-dimensional T1-weighted MRI sequences by a single researcher (MLS). The 3-dimensional tracings of the cerebellum were facilitated by semiautomated, interactive tools (http://rview.colin-studholme.net; version 9.071) with simultaneous display of axial, sagittal, and coronal views, in a method previously reported in Tam et $\mathrm{al}^{8}$ (Figure 1). This method shows good intrarater reliability (Dice coefficient $>0.95$ ) on repeat tracings.

Total cerebral volume was calculated at each time point using multiple automatically generated templates (MAGeT) brain. ${ }^{24}$ First, 
the gray and white matter and cerebrospinal fluid of 1 randomly selected MRI scan from each serial time point was manually segmented and nonlinearly registered to 21 MRIs of that serial scan time point using advanced normalization tools. ${ }^{25,26}$ The 21 MRIs and their labels of gray and white matter and cerebrospinal fluid were later employed as the input atlases. The same 21 images were used as intermediate templates in MAGeT-Brain. The atlases and templates were then used to segment the entire data set within each serial time point. The final segmentation of each MRI was obtained through the label fusion of 441 nonlinearly registered labels ( 21 atlases $\times 21$ templates), which limits segmentation errors resulting from resampling, nonlinear registration, or irreconcilable differences in neuroanatomy. Total cerebral volume (gray and white matter-excluding the cerebrospinal fluid in the lateral and third ventricles) of each MRI was derived individually based on the MAGeT-Brain segmentation results acquired from the previous step.

\section{Statistical Analysis}

Statistical analyses were performed using Stata 12 (Stata Corporation, College Station, TX). Descriptive statistics were used to analyze the characteristics of the study group, including the $t$ test for means, the Fisher exact test for proportions of binary variables, and the Kruskal-Wallis rank test for categorical variables. Linear regression analyses were used to examine the association between cerebellar volumes at each of the 3 analyzed time points, correcting for postmenstrual age at the time of each MRI scan. Linear regression analyses were also used to assess the association between cerebral volume at term and 2 years of age, correcting for postmenstrual age. Maximum parental education was categorized from 0 through 4 for grade school (0), high school (1), postsecondary training (2), university (3), and postgraduate training (4). Maternal and paternal education were also analyzed separately using these 5 categories and again with education further categorized as no postsecondary $(a)$ and postsecondary or above $(b)$. Linear regression analyses were also used to assess the associations with cerebral brain volumes at term and 2 years of age, correcting for age at the time of each scan. These were then adjusted for weight and head circumference at 2-year MRI to rule out nutritional factors for improved cerebellar growth. The association between maternal education and brain volumes at each time point was analyzed in order to rule out a predisposition for larger or smaller volume associated with maternal education.

\section{Results}

\section{Study Cohort}

Out of a cohort of 105 subjects enrolled during the study period, serial scans were obtained within 2 weeks of life and near term-equivalent age. Of these, 26 subjects also had MRI scans at 2 years of age (Figure 2). There were no significant differences in the demographics, clinical characteristics, or brain injury on early MRIs between enrolled subjects and those with completed MRIs to 2 years (Table 1). A total of 73 serial MRI scans from these 26 subjects were segmented, and volumetric data were analyzed. Infants were scanned at mean postmenstrual ages of $30.2 \pm 1.7$ weeks for the preterm scan, $41.9 \pm 2.0$ weeks for the term-equivalent age scan, and $151.8 \pm 6.7$ weeks for the 2-year scan.

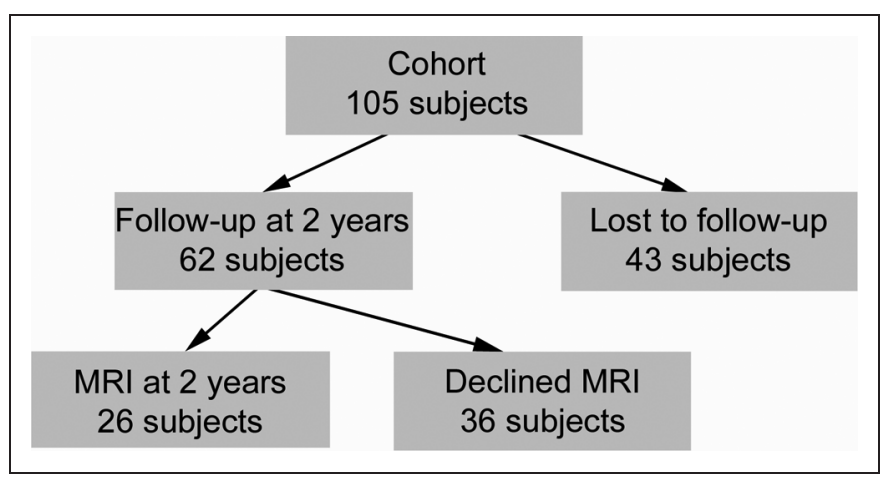

Figure 2. Flow diagram of study subjects. Subjects who returned for MRI scans at 2 years of age were included in the study analysis. Socioeconomic status was obtained at the 2-year follow-up visit.

Table I. Subject Demographics and Clinical Characteristics. ${ }^{a}$

\begin{tabular}{|c|c|c|c|}
\hline \multirow[b]{2}{*}{ Characteristic } & \multicolumn{2}{|c|}{$\begin{array}{l}\text { Two-year MRI } \\
\text { completed }\end{array}$} & \multirow[b]{2}{*}{$\begin{array}{c}P \\
\text { value }\end{array}$} \\
\hline & $\begin{array}{c}\text { Yes } \\
(n=26)\end{array}$ & $\begin{array}{c}\text { No } \\
(n=79)\end{array}$ & \\
\hline Male sex & $16(62)$ & $40(5 \mathrm{I})$ & .3 \\
\hline $\begin{array}{l}\text { Gestational age at birth } \\
\text { (mean } \pm S D), \text { wk }\end{array}$ & $28.9 \pm 1.7$ & $28.8 \pm 1.8$ & .8 \\
\hline Subjects with IVH & & & .7 \\
\hline Mild IVH (grade I to 2) & $4(15)$ & $16(20)$ & \\
\hline Severe IVH (grade 3 to 4 ) & $5(19)$ & $17(22)$ & \\
\hline Subjects with WMI & & & .8 \\
\hline Mild WMI (grade I) & $2(8)$ & $10(13)$ & \\
\hline Moderate WMI (grade 2) & $6(23)$ & II (I4) & \\
\hline Severe WMI (grade 3) & $3(12)$ & $10(13)$ & \\
\hline Subjects with cerebellar hemorrhage & $3(12)$ & $7(9)$ & .7 \\
\hline Sepsis (clinical or confirmed) & $18(69)$ & $4 \mid(52)$ & .1 \\
\hline $\begin{array}{l}\text { Chorioamnionitis (clinical or } \\
\text { confirmed) }\end{array}$ & $2(8)$ & $19(24)$ & .07 \\
\hline Antenatal steroid exposure & I5 (58) & $57(72)$ & .2 \\
\hline Postnatal steroid exposure & I (4) & $5(6)$ & .7 \\
\hline
\end{tabular}

Abbreviations: IVH, intraventricular hemorrhage; MRI, magnetic resonance imaging; SD, standard deviation; WMI, white matter injury.

${ }^{a} V$ alues are $\mathrm{n}(\%)$ unless otherwise noted.

\section{Cerebellar Volumes at Early Postnatal, Term, and 2-Year Time Points}

We compared the cerebellar volumes across serial scans for each subject. Adjusting for age at the time of each MRI scan, a significant association was seen between term cerebellar volume and 2-year cerebellar volume; a $1 \mathrm{~cm}^{3}$ smaller volume at term was associated with $3.18 \mathrm{~cm}^{3}$ smaller volume at 2 years ( $95 \%$ confidence interval $1.5-4.8 \mathrm{~cm}^{3}, P=.001$ ). Considering the mean cerebellar volume was $23.9 \mathrm{~cm}^{3}$ at term and 106.2 $\mathrm{cm}^{3}$ at 2 years, this translates to a $4 \%$ smaller volume at term associated with a $3 \%$ smaller volume at 2 years. Cerebellar volume in the preterm period was not significantly associated with 2-year cerebellar volume, adjusting for postmenstrual age at time of MRI scan $(P=.4)$. Adjusting for gestational age at 
Table 2. Parental Education.

\begin{tabular}{lccc}
\hline & \multicolumn{2}{l}{ 2-year MRI completed, $\mathrm{n}(\%)$} & \\
\cline { 2 - 3 } Parental education & Yes $(\mathrm{n}=26)$ & No $(\mathrm{n}=36)$ & P value \\
\hline Maximum maternal education & & & .8 \\
$\quad$ Grade school & $\mathrm{I}(4)$ & $0(0)$ & \\
High school & $4(15)$ & $6(17)$ & \\
Postsecondary training & $5(19)$ & $10(28)$ \\
University & $13(50)$ & $16(44)$ \\
Postgraduate training & $\mathrm{I}(4)$ & $\mathrm{I}(3)$ \\
Not reported & $2(8)$ & $3(8)$ \\
Maximum paternal education & & & .3 \\
Grade school & $\mathrm{I}(4)$ & $0(0)$ \\
High school & $4(15)$ & $6(17)$ \\
Postsecondary training & $6(23)$ & $6(17)$ \\
University & $12(46)$ & $15(42)$ \\
Postgraduate training & $\mathrm{I}(4)$ & $6(17)$ \\
Not reported & $2(8)$ & $3(8)$ \\
\hline
\end{tabular}

Abbreviation: MRI, magnetic resonance imaging.

birth, intraventricular hemorrhage, white matter injury, cerebellar hemorrhage, and postnatal steroid exposure had no significant effects on the relationship between term and 2-year cerebellar volume $(P>.2)$.

\section{Cerebellar Growth and Parental Education}

The association between parental education and cerebellar growth was assessed. Of the full cohort of 105 subjects, data on parental education are available for the $62(59 \%)$ who returned for follow-up Bayley developmental assessments at 2 years (including the 26 infants with MRI scans at 2 years; Figure 2). Three families declined to share education level, and 2 families did not report either maternal or paternal education level. No differences in maternal or paternal education were found between those who returned for clinical follow-up only and those who also consented to MRI assessments (Table 2). Considering only $11 \%$ of adult Toronto residents have not completed high school and $69 \%$ have postsecondary degrees, our study sample has somewhat higher proportion of families with postsecondary degrees but comparable proportion of families not completing high school. ${ }^{27}$ Maternal postsecondary education was a significant confounder in the association between term and 2-year cerebellar volume $\left(30.05 \mathrm{~cm}^{3}, 95 \%\right.$ confidence interval $6.72-53.38 \mathrm{~cm}^{3}, P=.016$ ), even after adjusting for weight and head circumference at 2-year assessment (30.95 $\mathrm{cm}^{3}, 95 \%$ confidence interval $\left.0.58-61.33 \mathrm{~cm}^{3}, P=.047\right)$. Paternal postsecondary education was not similarly associated either before $(P=.1)$ or after adjusting for weight and head circumference $(P=.2)$. Adjusting for maternal postsecondary education diminished the association between term and 2-year cerebellar volumes. Although $1 \mathrm{~cm}^{3}$ smaller volume at term was associated with a $3.18 \mathrm{~cm}^{3}$ smaller volume at 2 years (95\% confidence interval $1.55-4.82 \mathrm{~cm}^{3}, P=.01$ ) prior to adjusting for parental education, adjusting for maternal postsecondary education decreased the association to $2.40 \mathrm{~cm}^{3}$ smaller volume at 2 years $\left(95 \%\right.$ confidence interval $0-4.8 \mathrm{~cm}^{3}$, $P=.054)$. Adjusting for paternal postsecondary education, the association was not diminished $\left(3.31 \mathrm{~cm}^{3}, 95 \%\right.$ confidence interval $0.8-5.8 \mathrm{~cm}^{3}, P=.01$ ).

To evaluate whether maternal education is associated with generally larger cerebellar volumes, linear regression analysis was performed comparing postsecondary maternal education and cerebellar volume at term and 2 years. Maternal education at or exceeding postsecondary training was found to be associated with cerebellar volumes at 2 years corrected age $(P=.006)$, but not cerebellar volumes at term-equivalent age $(P=.16)$.

\section{Cerebral Volumes at Early Postnatal, Term, and 2-Year Time Points}

Adjusting for postmenstrual age at the time of each MRI scan, a significant association was seen between term cerebral volume and 2-year cerebral volume, with a $1 \mathrm{~cm}^{3}$ decrease in cerebral volume at term associated with a $2.2 \mathrm{~cm}^{3}$ decrease in cerebral volume at 2 years $\left(95 \%\right.$ confidence interval $1.4-2.9 \mathrm{~cm}^{3}$, $P<.001)$. Adjusting further for parental education, neither maternal $(P=.28)$ nor paternal $(P=.22)$ postsecondary education was a significant confounder for this association.

\section{Neurodevelopmental Outcome at 2 Years}

Of the 26 subjects with completed 2-year MRI studies, Bayley developmental assessments were performed at a mean of $148.2 \pm 16.3$ weeks' postmenstrual age. Mean cognitive score for the group was $113 \pm 16$, motor score $96 \pm 13$, and language score $100 \pm 16$. Scores were not significantly different from the remaining subjects who returned for 2-year developmental testing but declined MRI, where mean cognitive score was $114 \pm 26(P=.8)$, motor score $93 \pm 14(P=.4)$, and language score $100 \pm 15(P=1.0)$. Using linear regression analysis adjusting for age at MRI and developmental testing, neither cerebellar volume at term nor 2 years was associated with Bayley scores in any domain $(P>.3)$.

\section{Discussion}

The cerebellum is particularly vulnerable to impaired development in very preterm infants due to the exponential growth that occurs during the third trimester of gestation. ${ }^{3,4}$ Associated with preterm birth, cerebellar hypoplasia ${ }^{14}$ and low familial socioeconomic status ${ }^{16}$ have been associated with impairments in cognitive and motor performance; however, little is known about the connection between socioeconomic status and structural changes within the cerebellum. Using parental education as an indicator for socioeconomic status, we explored the relation between cerebellar growth and socioeconomic status in a longitudinal study from very preterm birth to 2 years of age.

Volumetric analyses of serial MRI scans revealed a predictive association between cerebellar volume at term-equivalent age and volume at 2 years of age. The same relationship did not hold true for the preterm cerebellar volume as a predictor 
of 2-year volume. This is likely due to volumetric changes associated with clinical factors, such as intraventricular hemorrhage and steroids, impacting growth over the early postnatal period and being evident only at term-equivalent age. ${ }^{8,28}$

Adjustment for parental education as a measure of socioeconomic status lessened the significance of the association between cerebellar volumes at the term-equivalent and 2-year time points. Adjusting for maternal postsecondary education in particular attenuated the relationship between term and 2-year cerebellar volumes resulting in improved volumes by 2 years of age. Higher levels of maternal education were not found to be correlated with larger cerebellums by term age, suggesting that the effect of maternal education does not lie in genetic resilience to insults in the early postnatal period but in different exposures after hospital discharge in families with higher education. Adjusting for body weight and head circumference did not attenuate the association between maternal education and cerebellar growth, suggesting that the mechanisms improving cerebellar growth are independent of factors affecting somatic growth, such as nutrition.

While brain injury after preterm birth has been well documented and studied in relation to impaired outcomes, the rapid growth of the cerebellum during the latter portion of gestation makes it particularly susceptible to adverse effects in preterm infants. Between 27 and 40 weeks of postmenstrual age, an infant's cerebellum increases 5 -fold in volume ${ }^{28}$ and even more so in surface area; cerebral growth is not as exponential during this time. Although the germinal matrix largely involutes by 32 weeks' gestational age in the cerebrum, the germinal matrix (external granular layer) of the cerebellum may not involute until the age of 2 years. ${ }^{29}$ For this reason, it was not surprising that the moderating effects of maternal postsecondary education did not impact cerebral growth as it did with cerebellar growth.

Our results in the cerebrum are also supported by a recent study of 77 children imaged serially from 5 months to 4 years from the US National Institute of Health MRI Study of Normal Brain Development, which showed no statistically significant difference in total cerebral volume associated with socioeconomic status categorized based on household income. ${ }^{30}$ Although total cerebral volume was not different, further separation into gray and white matter regions identified lower average frontal and parietal gray matter volumes associated with low socioeconomic status. Thus, although we did not find overall cerebral volume differences here, regional differences may be present.

The current study suggests that there are measurable structural correlates between socioeconomic status and cerebellar growth between term and 2 years of age. Because the late gestational and postnatal period is when the greatest cerebellar development and expansion takes place, external factors play a significant role in influencing the cerebellum in children born prematurely. ${ }^{3,5}$ Socioeconomic status is often a strong indicator of the environmental factors to which a child will be exposed to in early life. This study examined how one measure of socioeconomic status (parental education) compared to postnatal cerebellar growth in a cohort of preterm infants, assuming that higher levels of parental education correspond with a greater access to resources, better parenting skills, and enhanced cognitive stimulation. ${ }^{31-33}$

Splitting the parents of the cohort subset into those who had and had not pursued postsecondary education produced an attenuation of the predictive relationship between term volume and 2-year volume was observed for those with postsecondary training. This effect had greater significance when adjusting for maternal than paternal education. Although previous studies have shown an important role for paternal education as an indicator of risk for preterm birth ${ }^{34}$ and a better marker of a woman's health-related behaviors than her own socioeconomic status, ${ }^{35}$ the present study is unique as it focuses on postnatal growth. Maternal education appeared to be a better marker for postnatal changes to cerebellar volume than paternal education.

These results echo findings in an emerging body of literature regarding the impact of social adversity on cellular pathways that lead to worse outcomes in health and development. Social adversity has been found in animal and human studies to be associated with changes in epigenetics, inflammation, and endocrinological responses. Maternal behavior can induce epigenetic changes in the glutamate receptor gene to affect plasticity of the hippocampus in rat pups. ${ }^{36}$ In a study of both mice and humans, social stress upregulates inflammatory gene expression in white blood cells. ${ }^{37}$ In humans, lower maternal education was found to be associated with impairments in a child's neural response to sound. ${ }^{38}$ Parental stress and depression is associated with dysregulation of the child's hypothalamic-pituitary-adrenal axis..$^{39}$ Meanwhile, prenatal socioeconomic adversity is associated with epigenetic changes in placental steroid metabolism, ${ }^{40}$ which may affect brain development, and particular cerebellar development. ${ }^{41}$

This study was limited by the restricted socioeconomic status-related data collected from parents. In the future, collection of more detailed socioeconomic status data such as years of education, income, and occupational history may be valuable in better assessing the association between socioeconomic status and postnatal cerebellar growth in preterm neonates. Furthermore, neuroimaging of subjects at 2 years of age was a challenge, both in terms of feasibility and also parental consent, which resulted in a small sample size for analysis for this study. To address the issues surrounding loss to follow-up, available clinical (Table 1), socioeconomic status (Table 2), and developmental testing variables were compared. These did not show any significant differences between subjects who received MRI at 2 years and those who did not return for assessment.

Developmental assessment of this cohort at 2 years of age resulted in normal testing throughout the cohort, and no association with term cerebellar volumes. These results are in contrast with previous observed associations between term cerebellar volume and 2-year outcomes. ${ }^{6,42}$ The findings here may thus be limited by the small sample size. As well, further testing at later follow-up may elucidate more specific neurodevelopmental impairments.

Although preterm birth presents many known risk factors for impaired cerebellar development, our study findings suggest that supportive postnatal factors associated with high 
socioeconomic status may counteract these early clinical risk factors, resulting in improved cerebellar growth that may ultimately affect neurodevelopmental outcomes. These findings highlight not only the importance of identifying postnatal socioeconomic status factors in studies of neurodevelopmental outcome but also of the importance of ensuring good follow-up in both high and low socioeconomic status subjects. Studies with inadequate follow-up in low socioeconomic status subjects or lack of consideration of socioeconomic status may limit the ability to identify important associations between risk factors and outcome. With further investigation into the most influential factors affecting postnatal cerebellar growth, educational programs targeting in particular low socioeconomic status families may help reduce the adverse effects of preterm birth on cerebellar growth and development.

\section{Acknowledgments}

The authors thank Steven P. Miller, MDCM, MAS, FRCPC (Department of Pediatrics, Hospital for Sick Children, Toronto, Canada), and Donna M. Ferriero, MD, MS (Departments of Pediatrics and Neurology, University of California San Francisco), for reviewing the manuscript, and also to the families and children who participated in this research study. These results were presented at the Pediatric Academic Societies Annual Meeting in May 2014.

\section{Author Contributions}

MLS performed cerebellar analyses and data collection, and wrote the first draft of the manuscript. DK aided in the imaging analysis and data collection. TG and AT performed the cerebral analyses. AT aided in subject recruitment and clinical data collection. MJT led the recruitment and data collection of the study cohort. EWYT formulated the study question, performed data analysis, and interpreted study findings. All authors critically reviewed the manuscript and approved of the final version for submission.

\section{Declaration of Conflicting Interests}

The authors declared no potential conflicts of interest with respect to the research, authorship, and/or publication of this article.

\section{Funding}

The authors disclosed receipt of the following financial support for the research, authorship, and/or publication of this article: This work is supported by CIHR MOPC84399 [MJT]. The work of MLS was supported by the SickKids Summer Research Program.

\section{Ethical Approval}

This study was approved by the Research Ethics Board (approval number 1000038183) at the Hospital for Sick Children, and all infants were enrolled after informed consent was obtained from the parents or legal guardians.

\section{References}

1. Whitehead NS. The relationship of socioeconomic status to preterm contractions and preterm delivery. Matern Child Health J. 2012;16:1645-1656.

2. Martin JA, Hamilton BE, Ventura SJ, et al. Births: final data for 2011. National Vital Statistics Reports. 2013;62(1):1-69.
3. Limperopoulos C, Soul JS, Gauvreau K, et al. Late gestation cerebellar growth is rapid and impeded by premature birth. Pediatrics. 2005;115:688-695.

4. Gilmore JH, Schmitt JE, Knickmeyer RC, et al. Genetic and environmental contributions to neonatal brain structure: a twin study. Hum Brain Mapp. 2010;31:1174-1182.

5. Cavanagh J, Krishnadas R, Batty GD, et al. Socioeconomic status and the cerebellar grey matter volume. Data from a wellcharacterised population sample. Cerebellum. 2013;12:882-891.

6. Messerschmidt A, Fuiko R, Prayer D, et al. Disrupted cerebellar development in preterm infants is associated with impaired neurodevelopmental outcome. Eur J Pediatr. 2008; 167:1141-1147.

7. Srinivasan L, Allsop J, Counsell SJ, Boardman JP, Edwards AD, Rutherford M. Smaller cerebellar volumes in very preterm infants at term-equivalent age are associated with the presence of supratentorial lesions. AJNR Am J Neuroradiol. 2006;27: 573-579.

8. Tam EW, Chau V, Ferriero DM, et al. Preterm cerebellar growth impairment after postnatal exposure to glucocorticoids. Sci Transl Med. 2011;3:105ra105.

9. Messerschmidt A, Fuiko R, Prayer D, et al. Disrupted cerebellar development in preterm infants is associated with impaired neurodevelopmental outcome. Eur J Pediatr. 2008;167:1141-1147.

10. Mercy JA, Steelman LC. Familial influence on the intellectual attainment of children. Am Sociol Rev. 1982;47:532-542.

11. Bradley RH, Corwyn RF. Socioeconomic status and child development. Annu Rev Psychol. 2002;53:371-399.

12. Scarr S, Weinberg RA. The influence of "family background" on intellectual attainment. Am Sociol Rev. 1978;43:674-692.

13. DeGarmo DS, Forgatch MS, Martinez CR. Parenting of divorced mothers as a link between social status and boys' academic outcomes: unpacking the effects of socioeconomic status. Child Dev. 1999;70:1231-1245.

14. Allin M, Matsumoto H, Santhouse AM, et al. Cognitive and motor function and the size of the cerebellum in adolescents born very pre-term. Brain. 2001;124:60-66.

15. Limperopoulos C, Bassan H, Gauvreau K, et al. Does cerebellar injury in premature infants contribute to the high prevalence of long-term cognitive, learning, and behavioral disability in survivors? Pediatrics. 2007;120:584-593.

16. Ardila A, Rosselli M, Matute E, Guajardo S. The influence of the parents' educational level on the development of executive functions. Dev Neuropsychol. 2005;28:539-560.

17. Noble KG, Norman MF, Farah MJ. Neurocognitive correlates of socioeconomic status in kindergarten children. Dev Sci. 2005;8: 74-87.

18. Noble KG, Wolmetz ME, Ochs LG, Farah MJ, McCandliss BD. Brain-behavior relationships in reading acquisition are modulated by socioeconomic factors. Dev Sci. 2006;9:642-654.

19. Wild KT, Betancourt LM, Brodsky NL, Hurt H. The effect of socioeconomic status on the language outcome of preterm infants at toddler age. Early Hum Dev. 2013;89:743-746.

20. Noble KG, McCandliss BD, Farah MJ. Socioeconomic gradients predict individual differences in neurocognitive abilities. Dev Sci. 2007;10:464-480. 
21. Roberts E, Bornstein MH, Slater AM, Barrett J. Early cognitive development and parental education. Infant Child Dev. 1999;8: 49-62.

22. Raviv T, Kessenich M, Morrison FJ. A mediational model of the association between socioeconomic status and three-yearold language abilities: the role of parenting factors. Early Child Res Q. 2004;19:528-547.

23. Nossin-Manor R, Card D, Morris D, et al. Quantitative MRI in the very preterm brain: assessing tissue organization and myelination using magnetization transfer, diffusion tensor and $\mathrm{T}_{1}$ imaging. Neuroimage. 2013;64:505-516.

24. Chakravarty MM, Steadman P, van Eede MC, et al. Performing label-fusion-based segmentation using multiple automatically generated templates. Hum Brain Mapp. 2013;34:2635-2654.

25. Avants BB, Tustison NJ, Song G, Cook PA, Klein A, Gee JC. A reproducible evaluation of ANTs similarity metric performance in brain image registration. Neuroimage. 2011;54:2033-2044.

26. Avants BB, Epstein CL, Grossman M, Gee JC. Symmetric diffeomorphic image registration with cross-correlation: evaluating automated labeling of elderly and neurodegenerative brain. Med Image Anal. 2008;12:26-41.

27. National Household Survey. Education in Canada: attainment, field of study and location of study. Statistics Canada, 2011; 99-012-X2011001, http://www.statcan.gc.ca/

28. Tam EW, Miller SP, Studholme C, et al. Differential effects of intraventricular hemorrhage and white matter injury on preterm cerebellar growth. J Pediatr. 2011;158:366-371.

29. Donkelaar HJ, Lammens M, Wesseling P, Thijssen HO, Renier WO. Development and developmental disorders of the human cerebellum. J Neurol. 2003;250:1025-1036.

30. Hanson JL, Hair N, Shen DG, et al. Family poverty affects the rate of human infant brain growth. PLoS One. 2013;8:e80954.

31. Evans GW. The environment of childhood poverty. Am Psychol. 2004;59:77-92.

32. Braveman PA, Cubbin C, Egerter S, et al. Socioeconomic status in health research: one size does not fit all. JAMA. 2005;294: 2879-2888.
33. Hackman DA, Farah MJ, Meaney MJ. Socioeconomic status and the brain: mechanistic insights from human and animal research. Nat Rev Neurosci. 2010;11:651-659.

34. Blumenshine PM, Egerter SA, Libet ML, Braveman PA. Father's education: an independent marker of risk for preterm birth. Matern Child Health J. 2011;15:60-67.

35. Bartley M, Martikainen P, Shipley M, Marmot M. Gender differences in the relationship of partner's social class to behavioural risk factors and social support in the Whitehall II study. Soc Sci Med. 2004;59:1925-1936.

36. Bagot RC, Zhang YT, Wen X, et al. Variations in postnatal maternal care and the epigenetic regulation of metabotropic glutamate receptor 1 expression and hippocampal function in the rat. Proc Natl Acad Sci U S A. 2012;109(suppl 2):1720017207.

37. Powell ND, Sloan EK, Bailey MT, et al. Social stress up-regulates inflammatory gene expression in the leukocyte transcriptome via beta-adrenergic induction of myelopoiesis. Proc Natl Acad Sci U $S$ A. 2013;110:16574-16579.

38. Skoe E, Krizman J, Kraus N. The impoverished brain, disparities in maternal education affect the neural response to sound. $J \mathrm{Neu}$ rosci. 2013;33:17221-17231.

39. Palmer F, Anand KJ, Graff JC, et al. Early adversity, socioemotional development, and stress in urban 1-year-old children. J Pediatr. 2013;163:1733-1739.

40. Appleton AA, Armstrong DA, Lesseur C, et al. Patterning in placental 11-B hydroxysteroid dehydrogenase methylation according to prenatal socioeconomic adversity. PLoS One. 2013;8:e74691.

41. Heine VM, Rowitch DH. Hedgehog signalling has a protective effect in glucocorticoid-induced mouse neonatal brain injury through an 11betaHSD2-dependent mechanism. J Clin Invest. 2009;119:267-277.

42. Lind A, Parkkola R, Lehtonen L, et al. Associations between regional brain volumes at term-equivalent age and development at 2 years of age in preterm children. Pediatr Radiol. 2011;41: 953-961. 